\title{
Depresión y ansiedad durante el aislamiento obligatorio por el COVID-19 en Lima Metropolitana
}

\author{
Depression and anxiety during the mandatory isolation period due to COVID-19 in Lima \\ Metropolitan Area
}

Diego E. Prieto-Molinaria,*, Gianella L. Aguirre Bravo ${ }^{a}$, Inés de Pierola ${ }^{a}$, Giancarlo Luna Victoria-de Bona ${ }^{a}$, Lucía A. Merea Silva ${ }^{a}$, Caleb S. Lazarte Nuñez ${ }^{\text {, }}$ Karla A. Uribe-Bravo ${ }^{a}$, Ángel Ch. Zegarra ${ }^{a}$

${ }^{a}$ Facultad de Psicología, Universidad de Lima, Perú

\section{Resumen}

Antecedentes: la implementación de medidas de salud pública como la cuarentena o el aislamiento son estrategias útiles para el control de una enfermedad; no obstante, también traen consecuencias sobre la salud mental. Objetivos: describir la prevalencia de síntomas de ansiedad y depresión en adultos de Lima Metropolitana, durante el periodo de aislamiento social en respuesta a la pandemia del COVID-19. Método: se utilizaron ítems de la Escala de Ansiedad de Lima [EAL-20] y de la Escala de Psicopatología Depresiva [EPD-6] en una muestra de 565 adultos que residen en Lima Metropolitana durante el la prevalencia de síntomas relacionados a la ansiedad y depresión. Además, se encontraron diferencias en función del sexo, edad, nivel educativo, y la modalidad del trabajo o estudio. Conclusiones: el aislamiento obligatorio implica la prevalencia de síntomas de ansiedad y depresión en adultos de Lima Metropolitana, los cuales se manifiestan de diferentes formas al considerar variables sociodemográficas. Esto es congruente con estudios a nivel internacional, por lo que deberían considerarse al tomar decisiones ligadas a políticas públicas de salud.

Palabras clave: coronavirus; salud mental; pandemias; depresión; ansiedad.

Para citar este artículo:

Prieto-Molinari, D. E., Aguirre, G. L., Pierola, I. de, Victoria-de Bona, G. L., Merea, L. A., Lazarte, C. S., Uribe-Bravo, K. A., \& Zegarra, Á. C. (2020). Depresión y ansiedad durante el aislamiento obligatorio por el COVID-19 en Lima Metropolitana. Liberabit, 26(2), e425. https://doi.org/10.24265/liberabit.2020.v26n2.09 Estado de Emergencia Sanitaria. Resultados: se identificó

\begin{abstract}
Background: the implementation of public health measures such as quarantines and isolation is a useful strategy for disease control; nonetheless, said measures may also have consequences on mental health. Objectives: to describe the prevalence of depression and anxiety symptoms in adults living in Lima Metropolitan Area during the social isolation period due to the COVID-19 pandemic. Method: items from the Lima Anxiety Scale [EAL-20] and the Depressive Psychopathology Scale [EPD-6] were used in a sample of 565 adults living in Lima Metropolitan Area during the health emergency. Results: prevalence of anxiety and depression symptoms were identified. Also, differences regarding sex, age, education level, and modality of work or study were found. Conclusions: the mandatory isolation period involves the prevalence of anxiety and depression symptoms in adults living in Lima Metropolitan Area. Such phenomena might occur in different ways according to the different sociodemographic variables. These findings are consistent with those from international studies; thus, they should be considered when making decisions related to public health policies.
\end{abstract}

Keywords: coronavirus; mental health; pandemics; depression; anxiety.

Este es un artículo Open Access publicado bajo la licencia Creative Commons Atribución 4.0 Internacional. (CC-BY 4.0)

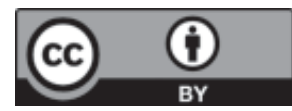

Universidad de San Martín de Porres, Lima - Perú

*depm_96@outlook.com 


\section{Introducción}

La aplicación de cuarentenas o de aislamiento social como medida de salud pública ha sido respaldada por la Organización Mundial de la Salud (OMS) durante el brote del COVID-19 (OMS, 2020). La Comisión Económica para América Latina y El Caribe (CEPAL) explica que las medidas de salud pública adoptadas para frenar el brote del COVID-19 pueden tener un fuerte impacto sobre la economía a nivel mundial y a nivel regional; lo cual podría llegar a causar la mayor crisis económica y social de la región en décadas (CEPAL, 2020). Más allá de las consecuencias económicas, las políticas de cuarentena y aislamiento pueden generar distintos estresores que deriven en afecciones de la salud mental (Brooks et al., 2020) manifestados tanto en las personas en cuarentena como en el personal encargado del cuidado de la salud (Hossain et al., 2020; Shuai et al., 2020). Brooks et al. (2020) hacen un resumen de las consecuencias sobre la salud mental que tuvo el brote del SARS. Ellos explican que el personal médico mostraba síntomas similares a los del trastorno por estrés agudo, los cuales se mantuvieron incluso después de tres años. Tras este lapso, la cuarentena se relacionaba con la frecuencia de abuso de alcohol, síntomas de dependencia y conductas de evitación. Asimismo, los niños en cuarentena y sus padres mostraron mayor prevalencia de trastorno por estrés postraumático (TEPT) y síntomas ligados al trauma (Brooks et al., 2020). Durante la epidemia generada por el MERS, se encontró mayor riesgo de depresión y de síntomas postraumáticos en pacientes con niveles elevados de angustia (Hyun-Chung et al., 2018).

En general, hay distintos factores que pueden aumentar o disminuir el riesgo de desarrollar síntomas de enfermedades mentales como producto de la exposición prolongada a estímulos estresores. Principalmente, deben tenerse en cuenta las diferencias que pueden encontrarse según distintas características sociodemográficas. Por ejemplo, durante la actual pandemia, se ha encontrado mayor nivel de ansiedad y depresión en trabajadoras de salud (HuarcayaVictoria, 2020; Pappa et al., 2020). Özdin y Özdin
(2020) han evidenciado que un factor de riesgo para el desarrollo de depresión es ser del género femenino y vivir en áreas urbanas. Por otro lado, se ha hallado que los jóvenes y la población con enfermedades crónicas muestran mayor nivel de depresión (OzamizEtxebarria et al., 2020). En estudiantes universitarios se han encontrado niveles de ansiedad y depresión más elevados que el nivel normativo nacional (Wang et al., 2020). El nivel educativo también ha mostrado ser una variable de importancia. Se ha encontrado que la prevalencia de depresión y ansiedad es mayor cuando la cantidad de años de escolaridad es menor, sobre todo en mujeres, y cuando las estrategias de afrontamiento son ineficaces para el manejo del estrés (Oliveira et al., 2020; Sinha \& Manna, 2020). Por último, el aislamiento social ha cambiado la forma en la que se realizan las actividades laborales y académicas. La educación virtual durante la pandemia puede generar sentimientos de soledad e insatisfacción que deriven en depresión (Misirlis et al., 2020). Por otro lado, el aumento de demandas familiares o la ausencia de habilidades tecnológicas para las conductas laborales se relacionan con mayor prevalencia de síntomas depresivos (Cho, 2020).

Las medidas establecidas por estados gubernamentales tienen como objetivo frenar la proliferación del COVID-19 y sus efectos colaterales. Entre ellos, uno de los aspectos más relevantes es el impacto que implica la pandemia sobre el estado de la salud mental de las personas. Rubin y Wessely (2020) predijeron, al inicio del brote de COVID-19 en Wuhan, niveles elevados de ansiedad en los ciudadanos. Asimismo, dentro de la comunidad científica se han comenzado a revisar las consecuencias sobre la salud mental que se dieron en epidemias y pandemias anteriores. Tavares et al. (2020) explican que la proliferación de una enfermedad puede conllevar a un aumento de la ansiedad en la comunidad, lo cual puede afectar las medidas de prevención que se han de tomar. Tras la confirmación del arribo del COVID-19 a Japón, se elevó la preocupación de que surjan distorsiones en la percepción de riesgo, síntomas de estrés, trastornos 
de salud mental ligados a la ansiedad y la depresión, y menor percepción de salud (Shigemura et al., 2020). En 2015, durante el brote del MERS-nCoV en Corea, se encontró que los individuos sin diagnóstico en cuarentena mostraron prevalencia de $7.6 \%$ y $16.6 \%$ de ansiedad y sentimientos de enojo. Entre cuatro y seis meses después, la prevalencia fue de $3 \%$ y $6.4 \%$, respectivamente (Park \& Park, 2020). En base a la proliferación del SARS en Taiwán, se encontraron efectos negativos en la salud mental del personal de cuidado de la salud: a medida que aumentaban los enfermos y muertos, aumentaron los niveles de ansiedad y disminuyó la disposición a trabajar (Schwartz et al., 2020). Se sugiere atender la salud mental de las personas infectadas, los contactos cercanos, los casos no confirmados en aislamiento, familia y amigos de las personas afectadas por el brote, y profesionales de la salud. No obstante, en situaciones de desastre biológico, el miedo, la incertidumbre y la estigmatización son comunes y pueden ser barreras para una apropiada intervención médica y de salud mental (Xiang et al., 2020). Las investigaciones que se han ido realizando en los últimos meses están confirmando estas preocupaciones en distintos países (OzamizEtxebarria et al., 2020; Özdin \& Özdin, 2020; Sinha \& Manna, 2020). No obstante, el contexto nacional carece de estudios que confirmen o contrasten los resultados hallados en otros países sobre el efecto de la pandemia en la salud mental. Por estos motivos, la presente investigación pretende cubrir este vacío de información como una aproximación exploratoria y descriptiva al estado de la salud mental a nivel nacional, particularmente, al describir la prevalencia de síntomas de ansiedad y depresión en adultos de Lima Metropolitana durante el periodo de aislamiento social obligatorio promulgado en el Perú como medida de salud pública en respuesta a la pandemia del COVID-19. En ese sentido, las comparaciones que se realizarán serán en base a lo planteado por otras investigaciones, pero no se plantea una postura teórica con hipótesis en tanto que no es el objetivo de esta exploración.

\section{Método}

\section{Participantes}

La presente investigación es un estudio descriptivo no experimental y transversal sobre características relacionadas a la sintomatología de ansiedad y depresión en una población de adultos residentes de Lima Metropolitana. Ello debido a que esta región presenta el mayor número de casos confirmados con diagnóstico de COVID-19 a nivel nacional. La estrategia de muestreo utilizada fue no probabilística y por conveniencia, la cual es usualmente utilizada en la investigación en ciencias sociales (Howitt \& Cramer, 2017). La muestra estuvo conformada por 565 adultos (382 mujeres y 183 hombres) que residían en Lima Metropolitana durante el Estado de Emergencia Sanitaria. En la Tabla 1 se puede encontrar información detallada sobre la composición de la muestra.

\section{Instrumentos}

Cuestionario de datos sociodemográficos. Se generó una sección para la recolección de información ligada a características sociodemográficas. Estas incluyeron la edad, sexo, nivel educativo máximo alcanzado, distrito de residencia, presencia de enfermedad crónica previa a la pandemia, y modalidad de trabajo o estudio.

Escala de Ansiedad [EAL-20] (Lozano-Vargas \& Vega-Dienstmaier, 2018). Se utilizaron 12 ítems del EAL-20, presentados en un formato de respuesta dicotómico (sí y no). Particularmente, se consideraron indicadores referidos a síntomas psíquicos y físicos, y otros relacionados a la agorafobia. Los puntajes obtenidos a través de la escala completa, compuesta por 20 ítems, muestran un coeficiente de confiabilidad de .889 a través del Alfa de Cronbach. Los puntajes obtenidos con esta escala cuentan con abcROC de $.757 \mathrm{y}$, en base al punto de corte sugerido ( $\geq 10$ ), la sensibilidad es de $69.4 \%$ y la especificidad es de $67.5 \%$. 


\section{Escala de Psicopatología Depresiva [EPD-6]} (Vega-Dienstmaier, 2018). Esta escala fue construida como una versión resumida de la Escala de Psicopatología Depresiva (EPD) para ser utilizada en tamizaje. El contenido de sus ítems corresponde a distintos síntomas propios de la depresión y son puntuados en una escala de Likert de cuatro categorías. Los puntajes de esta escala han mostrado un índice de confiabilidad de .736 a través del Alfa de Cronbach. Por otro lado, ha mostrado evidencias de validez en relación a otros cuestionarios de depresión con mayor cantidad de reactivos: coeficiente de Pearson con EPD-20 $=.889 \mathrm{y}$ coeficiente de Pearson con EDZ $=.764$. Los puntajes obtenidos con esta escala cuentan con un abcROC de .861 y, como prueba de tamizaje, el punto de corte sugerido $(\geq 8)$ cuenta con una sensibilidad de $80.2 \%$ y especificidad de $73.7 \%$.

\section{Procesamiento de los datos}

Inicialmente, se estimó el porcentaje de casos que presenta indicadores de ansiedad y depresión, considerando la frecuencia de respuestas individuales a cada ítem. Posteriormente, se desarrolló un análisis de las propiedades psicométricas obtenidas como resultado del proceso de evaluación con ambos instrumentos de medición. Para obtener evidencias de validez en relación a la estructura interna, se utilizó un Análisis Factorial Confirmatorio (AFC), estrategia ampliamente recomendada para este propósito. Este procedimiento se ejecutó en el software libre $R$ (versión 4.0.1) y con el paquete lavaan (versión 0.66). Además, se utilizaron matrices de correlaciones policóricas y el estimador $W L S M V$ para la implementación, en concordancia con las recomendaciones en la literatura sobre el uso del CFA en datos categóricos (Brown, 2015). El ajuste de los modelos unidimensionales para ambas escalas se juzgó a partir de los puntos de corte sugeridos para los indicadores Comparative Fit Index (CFI >.90), Root Mean Square Error of Approximation (RMSEA $<.08)$ y Standardized Root Mean Square Residual (SRMR <.08). A partir de las cargas factoriales estimadas, se calculó el coeficiente Omega (Viladrich et al., 2017). Este coeficiente permite aportar evidencias de la confiabilidad de las puntuaciones en relación a la consistencia interna; considerándose como apropiados los valores superiores a .70 .

Al haber obtenido propiedades psicométricas satisfactorias, se analizaron las variables del estudio a partir de estadísticos descriptivos segregados por las variables sociodemográficas sexo, edad, nivel educativo, modalidad de trabajo/estudio $y$ presencia de enfermedad de riesgo. Al observar la presencia de múltiples valores extremos en varias distribuciones, se procedió a emplear estadísticos robustos ante estos casos; específicamente, estrategias no paramétricas para la comparación entre grupos. Para variables de dos grupos se empleó la U de MannWhitney; y, para múltiples grupos, la $\mathrm{H}$ de KruskalWallis. La U de Mann-Whitney también fue utilizada como prueba post-hoc para complementar la $\mathrm{H}$, pero considerando la corrección de Bonferroni sobre el nivel de significancia de .05 para controlar el error Tipo I producto de implementar múltiples pruebas de significancia. El tamaño del efecto en cada comparación fue estimado a partir de la correlación rango-biserial, la cual puede interpretarse según las sugerencias de Cohen (1992). Todas las comparaciones fueron realizadas con el programa JASP (versión 0.12.2), un software libre con amplias herramientas para la investigación en ciencias sociales.

\section{Resultados}

\section{Presencia de indicadores de ansiedad y depresión}

En la figura 1 se presentan la frecuencia de indicadores de ansiedad en adultos de Lima Metropolitana. En general, por lo menos un $10 \%$ de los individuos refieren presentar el indicador respectivo. Sin embargo, en todos los indicadores hay una prevalencia menor al $50 \%$ de casos, con excepción del indicador referido a la incomodidad con respecto a entrar o salir de un lugar lleno de gente. 


\section{Figura 1}

Prevalencia de indicadores de ansiedad

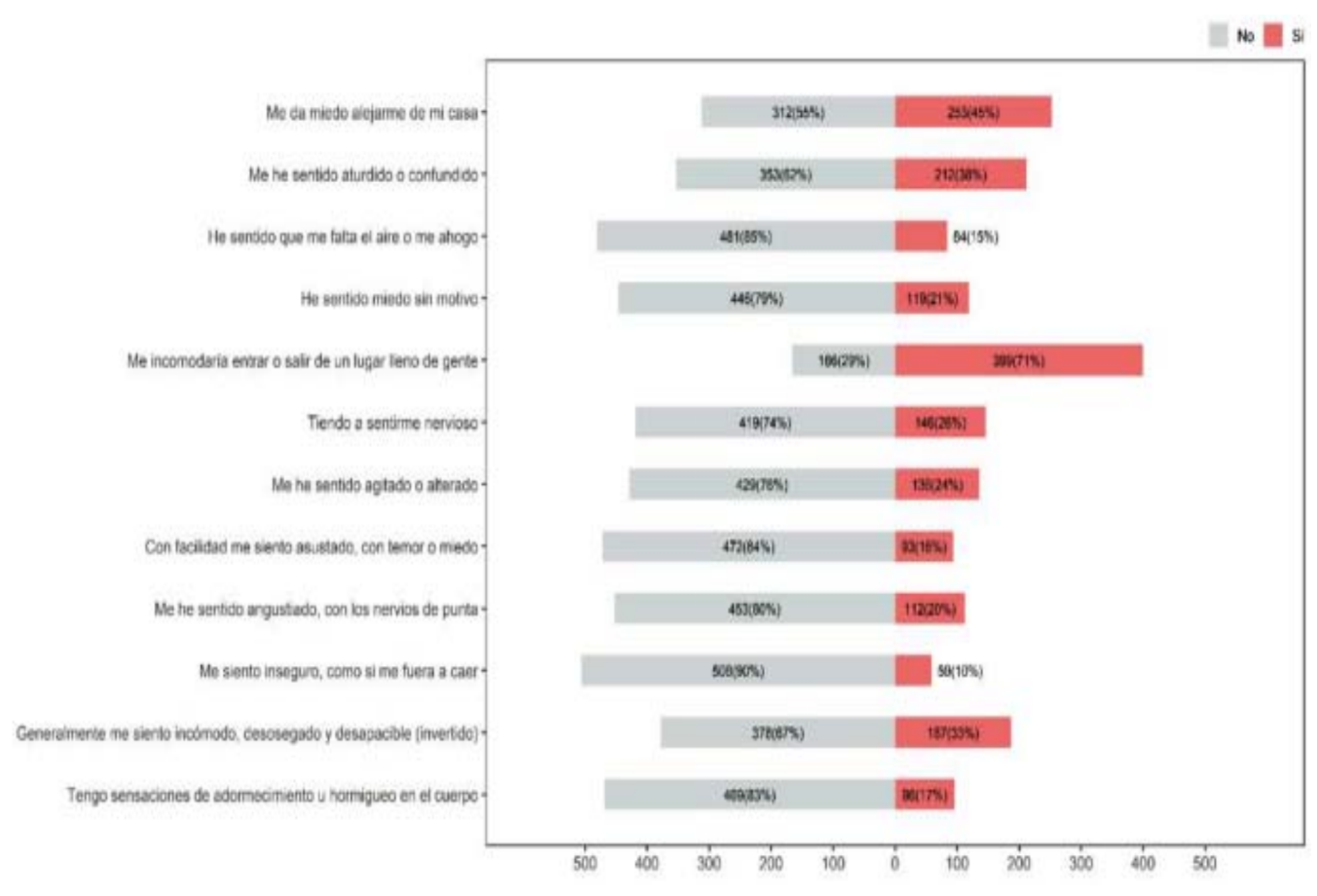

Figura 2

Prevalencia de indicadores de depresión

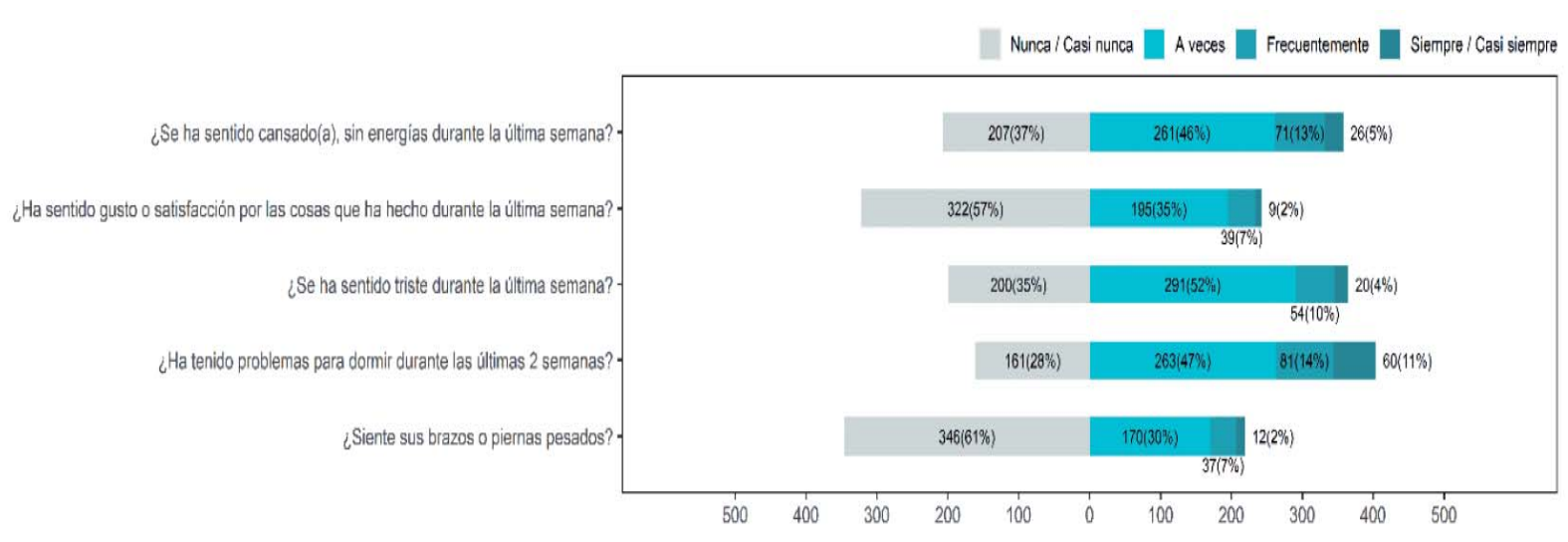

Los indicadores referidos a la sintomatología depresiva se presentan en la figura 2. A diferencia del caso anterior, por lo menos un $39 \%$ de individuos refiere presentar dichos síntomas. Particularmente, el indicador con mayor prevalencia fue la presencia de problemas para conciliar el sueño en $72 \%$ de individuos. 


\section{Propiedades psicométricas de las escalas}

Tras contrastar los resultados del AFC en las pruebas de ansiedad $\left(\chi^{2}[54]=141.011, p<.001\right.$, CFI $=.966, \mathrm{RMSEA}=.053$, IC 90\% [.043, .064], SRMR $=.070)$ y depresión $\left(\chi^{2}[5]=16.309, p<.006, \mathrm{CFI}=\right.$ .990, RMSEA $=.063$, IC 90\% [.031, .099], SRMR $=$ .034) con los puntos de corte propuestos por Keith (2019); los índices de ajuste señalaron que los modelos unidimensionales propuestos se ajustan apropiadamente a los datos empíricos en la muestra de adultos de Lima Metropolitana. Es importante mencionar que, como los tamaños de la muestra en determinados grupos sociodemográficos no fueron suficientes, no fue posible realizar un análisis de invarianza de la medición considerando ambos constructos. Por lo tanto, los resultados de las comparaciones entre grupos deben ser tomados con precaución. En otra instancia, los resultados de la estimación del coeficiente Omega demuestran un grado apropiado de confiabilidad en las puntuaciones de la escala de ansiedad $(w=.91) \mathrm{y}$ depresión $(w=.82)$, para su uso en investigación básica.

\section{Comparación según variables sociodemográficas}

En respuesta al objetivo de la investigación, se emplearon análisis descriptivos para analizar los niveles de ansiedad y depresión reportados en la muestra de participantes, segregando en base a distintas variables sociodemográficas para permitir la comparación de ambos fenómenos.

Tabla 1

Ansiedad y depresión en función a variables sociodemográficas

\begin{tabular}{|c|c|c|c|c|c|c|c|c|c|c|}
\hline \multirow{2}{*}{$\begin{array}{c}\text { Características } \\
\text { sociodemográficas }\end{array}$} & \multirow[t]{2}{*}{$n$} & \multirow[t]{2}{*}{$p$} & \multicolumn{4}{|c|}{ Ansiedad } & \multicolumn{4}{|c|}{ Depresión } \\
\hline & & & $M$ & $D E$ & $E E$ & $M d n$ & $M$ & $D E$ & $E E$ & $M d n$ \\
\hline $\begin{array}{l}\text { Total } \\
\text { Sexo }\end{array}$ & 565 & 1.0 & 3.36 & 2.77 & .12 & 3.00 & 3.76 & 2.80 & .12 & 3.00 \\
\hline Hombre & 183 & .32 & 2.26 & 2.13 & .16 & 2.00 & 3.03 & 2.38 & .18 & 3.00 \\
\hline Mujer & 382 & .68 & 3.88 & 2.89 & .15 & 3.00 & 4.11 & 2.92 & .15 & 3.00 \\
\hline \multicolumn{11}{|l|}{ Edad } \\
\hline Joven (18 a 29) & 305 & .54 & 3.83 & 2.94 & .17 & 3.00 & 4.39 & 2.76 & .16 & 4.00 \\
\hline Adulto (30 a 59) & 214 & .38 & 2.91 & 2.48 & .17 & 2.00 & 3.15 & 2.79 & .19 & 3.00 \\
\hline Adulto mayor (60 a más) & 46 & .08 & 2.28 & 2.34 & .34 & 2.00 & 2.46 & 1.93 & .28 & 2.00 \\
\hline \multicolumn{11}{|l|}{ Nivel educativo } \\
\hline $\begin{array}{l}\text { e incompleta } \\
\text { Superior universitaria }\end{array}$ & 70 & .12 & 4.44 & 3.33 & .40 & 3.00 & 4.47 & 3.21 & .38 & 4.00 \\
\hline $\begin{array}{l}\text { incompleta } \\
\text { Superior universitaria }\end{array}$ & 226 & .40 & 3.92 & 2.86 & .19 & 3.00 & 4.59 & 2.90 & .19 & 4.00 \\
\hline completa & 269 & .48 & 2.88 & 2.30 & .14 & 3.00 & 2.60 & 2.31 & .14 & 2.00 \\
\hline \multicolumn{11}{|l|}{ Modalidad de trabajo/estudio } \\
\hline Presencial & 126 & .22 & 2.92 & 2.52 & .23 & 2.00 & 3.05 & 2.07 & .18 & 3.00 \\
\hline Virtual o no trabaja/estudia & 439 & .78 & 3.48 & 2.83 & .14 & 3.00 & 3.97 & 2.95 & .14 & 3.00 \\
\hline \multicolumn{11}{|l|}{ Enfermedad de riesgo } \\
\hline $\begin{array}{l}\text { Por lo menos una enfermedad } \\
\text { No presenta enfermedades }\end{array}$ & 142 & .25 & 3.44 & 2.50 & .21 & 3.00 & 4.21 & 2.97 & .25 & 3.50 \\
\hline de riesgo & 423 & .75 & 3.33 & 2.86 & .14 & 3.00 & 3.61 & 2.73 & .13 & 3.00 \\
\hline
\end{tabular}

Nota: $n=$ número de casos, $p=$ proporción de casos, $M=$ media aritmética, $D E=$ desviación estándar, $E E=$ error estándar de la media aritmética, $M d n=$ mediana. 


\section{Ansiedad según sociodemográficas}

La comparación de los niveles de ansiedad en función a distintas variables sociodemográficas se presenta en la Figura 1. Con respecto al análisis de acuerdo al sexo, se encontraron diferencias estadísticamente significativas entre las puntuaciones obtenidas por hombres $(M d n=2.00)$ y mujeres $(M d n$ = 3.00), $\mathrm{U}=27478, p<.001$ con un tamaño del efecto mediano $(\mathrm{r}=-.36)$.

Del mismo modo, se encontraron diferencias estadísticamente significativas en los niveles de ansiedad según grupo de edad $(\mathrm{H}(2)=21.28$, $\mathrm{p}<$ .001). Particularmente, las pruebas a posteriori revelaron diferencias pequeñas al comparar a adultos $(M d n=2.00)$ con jóvenes $(M d n=3.00)(U=$ 26891.5, $p=.001, r=-.18)$ y diferencias medianas al comparar adultos mayores $(M d n=2.00)$ con jóvenes $(U=4688.5, p=.001, r=-.33)$. En contraste, no se encontraron diferencias estadísticamente significativas al comparar a adultos con adultos mayores $U=5886.5, p=.034, r=.0196$, considerando un valor crítico de 0.17 .

También se encontraron diferencias estadísticamente significativas de acuerdo al nivel educativo de $H(2)=36.68, p<.001$. De modo específico, al comparar a personas con educación técnica completa o incompleta $(M d n=3.00)$ con aquellas con educación universitaria completa ( $M d n$ = 3.00), $U=38718.5, p<.001$, con un tamaño del efecto pequeño $r=.274$. Y, al comparar a personas con educación universitaria incompleta $(M d n=3.00)$ con aquellas que finalizaron dicha etapa académica $U=12586, p<.001$, pero en este caso, las diferencias tienen un tamaño del efecto mediano $r=$ .34. No se encontraron diferencias entre personas con educación técnica completa o incompleta con aquellos con educación universitaria incompleta $U=$ 8503, $p<.340, r=-.08$.

En otra instancia, los análisis con respecto a la modalidad de trabajo o estudio encontraron diferencias estadísticamente significativas entre los niveles de ansiedad de las personas que trabajan o estudian de manera presencial durante el periodo de aislamiento $(M d n=2.00)$ frente a aquellas que no ( $M d n=3.00), U=30959, p=.039$, aunque con un tamaño del efecto pequeño $r=.12$.

Finalmente, no se encontraron diferencias estadísticamente significativas entre las personas que padecen por lo menos una enfermedad de riesgo ante el covid-19 (enfermedades cardiovasculares, diabetes, hepatitis $\mathrm{B}$, asma, enfermedades crónicas renales o cáncer; $M d n=3.00$ ) y aquellas que no padecen ninguna de estas enfermedades $(M d n=3.00), U=$ 27937.5, $p=.209, r=-.07$.

\section{Depresión según características sociodemográficas}

La comparación de los niveles de depresión en función a distintas variables sociodemográficas se presenta en la Figura 2. Al comparar este fenómeno en función al sexo de las personas, se encontraron diferencias estadísticamente significativas entre las puntuaciones obtenidas por hombres $(M d n=3.00)$ y mujeres $(M d n=3.00), U=22491.5, p<.001$, pero con un tamaño del efecto pequeño $(r=-.21)$.

Los resultados del análisis con respecto al grupo de edad indican diferencias estadísticamente significativas en los indicadores de depresión reportados por dichos grupos etarios: $H(2)=49.29$, $p<.001$. Específicamente, estas diferencias se encuentran al comparar a adultos $(M d n=3.00)$ con jóvenes $(M d n=4.00)(U=22670.5, p<.001) \mathrm{y}$ adultos mayores $(M d n=2.00)$ con jóvenes $(U=$ 3844, $p<.001)$. Además, en ambos casos el tamaño del efecto indica una significancia práctica mediana $r=-.31$ y $r=-.45$, respectivamente. En contraste, no se encontraron diferencias al comparar a adultos con adultos mayores $U=5598, p=.139, r=.137$.

En relación a al nivel educativo, se encontraron diferencias estadísticamente significativas en los niveles de depresión de las personas: $(H(2)=55.08$, 
$p<.001)$. Las pruebas a posteriori demostraron que dichas diferencias se encuentran al comparar a personas con educación técnica completa o incompleta $(M d n=4.00)$ con aquellas con educación universitaria completa $(M d n=2.00)(U=41740.5$, $p<.001$ ), con un tamaño del efecto mediano $r=.37$. Un resultado similar se encuentra al comparar a personas con educación universitaria incompleta $(M d n=4.00)$ con aquellas que finalizaron dicha etapa académica $(U=12246, p<.001, r=.30)$. No obstante, no se encontraron diferencias entre personas con educación técnica completa o incompleta con aquellos con educación universitaria incompleta $(U=7492.5, p=.502, r=-.05)$.

En función a la modalidad de trabajo o estudio, se encontraron diferencias estadísticamente significativas entre los niveles de depresión de las personas que trabajan o estudian de manera presencial durante el periodo de aislamiento $(M d n=$ $3.00)$ frente a aquellas que no $(M d n=3.00), U=$ 32188, $p=.005$, aunque con un tamaño del efecto pequeño $r=.16$.

Finalmente, se encontraron diferencias estadísticamente significativas en los niveles de depresión en función a la presencia de una enfermedad de riesgo ( $U=27937.5, p=.209$ ). Sin embargo, las diferencias entre aquellas personas que padecen por lo menos una enfermedad de riesgo ante al covid-19 (enfermedades cardiovasculares, diabetes, hepatitis B, asma, enfermedades crónicas renales o cáncer) (Mdn $=3.50)$ y aquellas que no padecen ninguna de estas enfermedades $(M d n=3.00)$ es pequeña $(r=-.12)$.

\section{Figura 3}

Comparación de los niveles de ansiedad en función a variables sociodemográficas
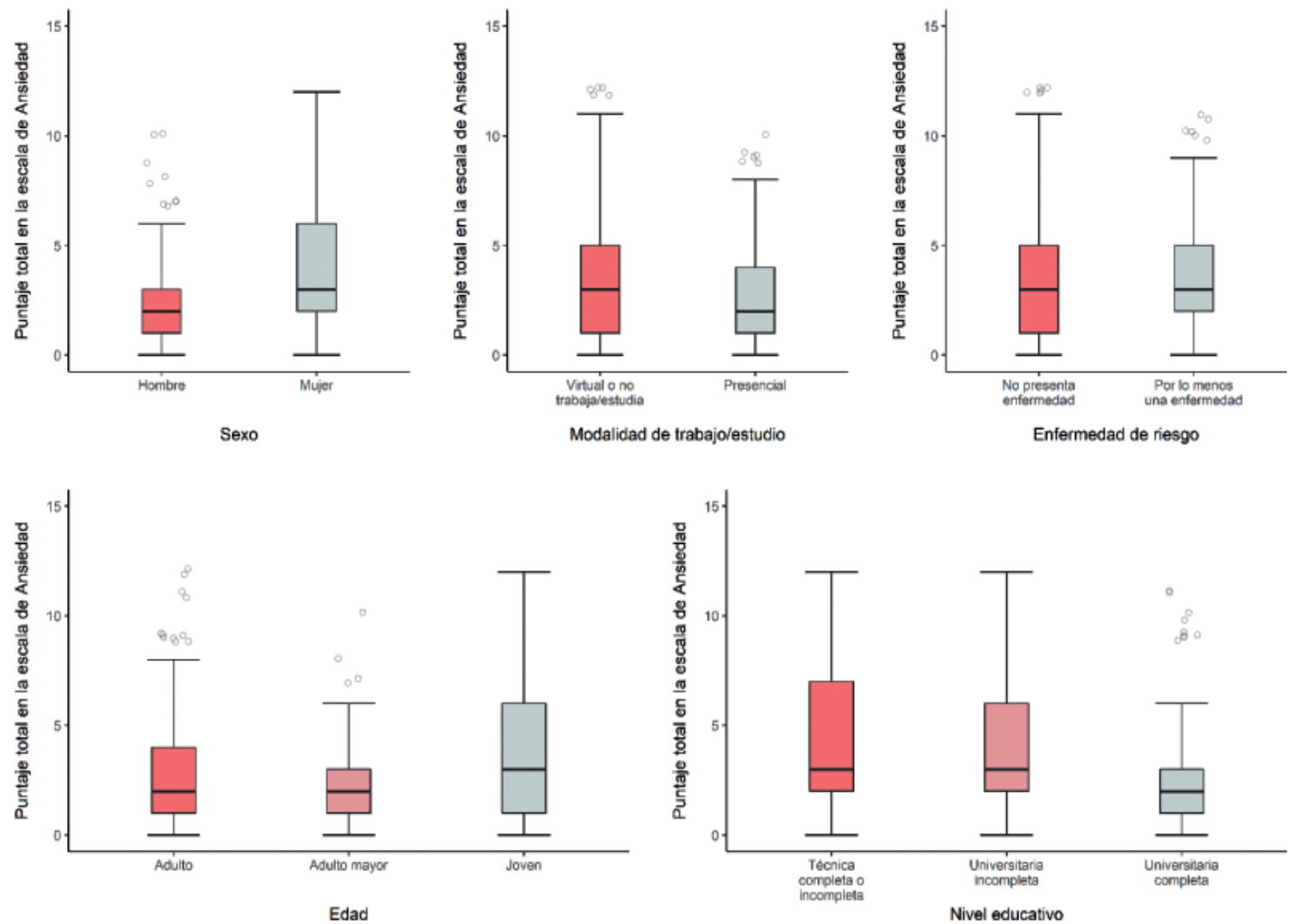
Figura 4

Comparación de los niveles de depresión en función a variables sociodemográficas
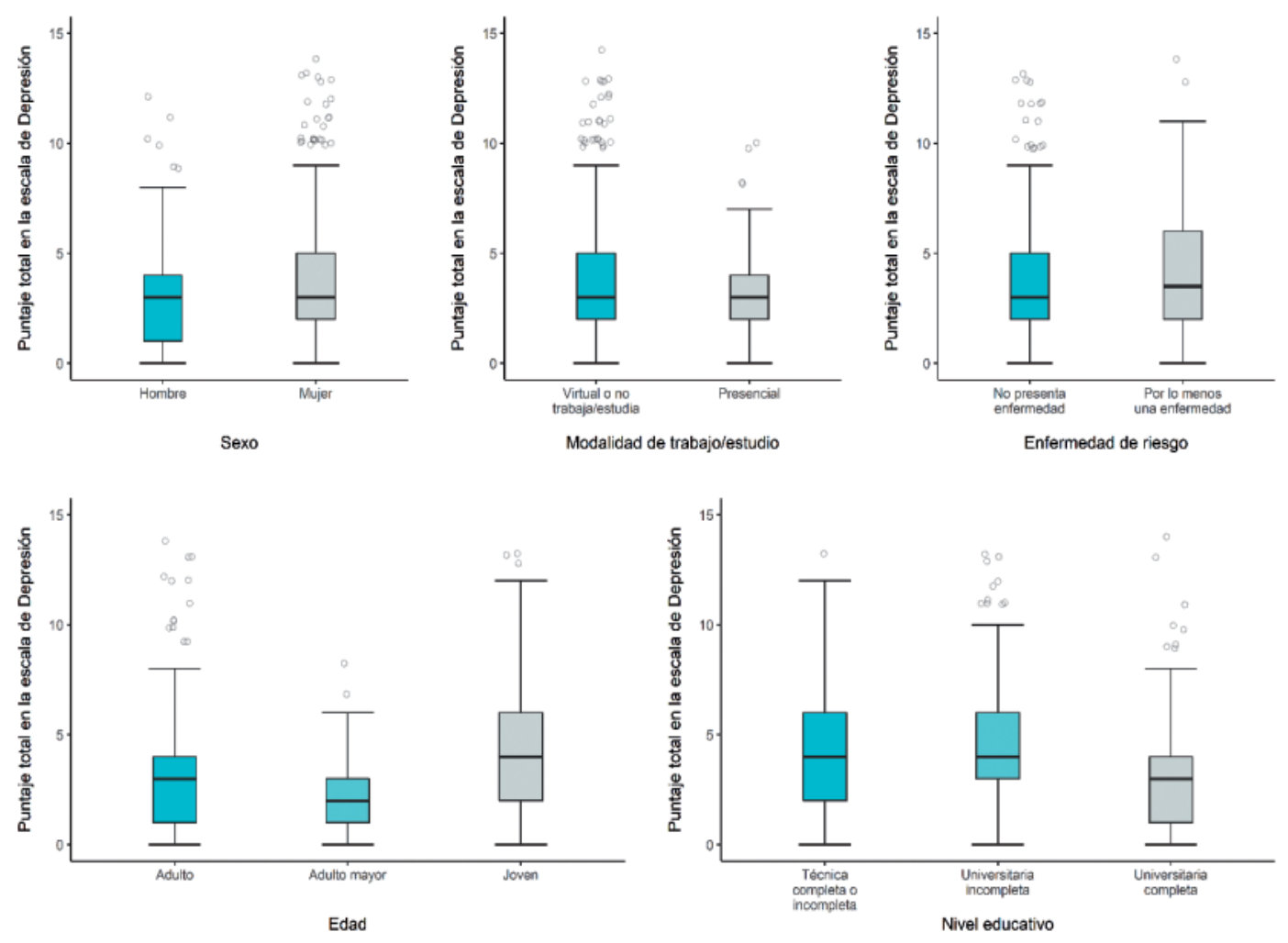

\section{Discusión}

La presente investigación tuvo como objetivo describir los niveles de ansiedad y depresión en adultos de Lima Metropolitana durante el periodo de aislamiento social obligatorio. Ciertamente, la literatura internacional comprende varios estudios sobre esta temática, los cuales guían la toma de decisiones por parte de las instituciones gubernamentales en relación a cómo enfrentar la pandemia. No obstante, aunque los estudios internacionales puedan brindar información importante y empírica sobre el fenómeno, es necesario un estudio contextualizado que permita describir con mayor precisión el estado de diversas características de la salud mental a nivel nacional. Por esta razón, este estudio se presenta como una aproximación exploratoria al abordaje contextualizado de características de la salud mental en la región de Lima Metropolitana.

Principalmente, se detectó la prevalencia de varios síntomas relacionados a la ansiedad y depresión, hallazgo esperado y congruente con la mayoría de los estudios contemporáneos en esta misma línea de investigación (Brooks et al., 2020; Huarcaya-Victoria, 2020). Particularmente, los indicadores de ansiedad con mayor prevalencia son características representativas de la agorafobia, por ejemplo, la incomodidad al encontrarse en un lugar muy concurrido o, incluso, al temor a salir de casa. En efecto, estudios internacionales reportan el mismo resultado aludiendo a que, como el contacto con otras personas involucra una potencial fuente de contagio, es natural que dicho temor o incomodidad sea común. En otra instancia, los síntomas depresivos con mayor prevalencia se encuentran referidos a los problemas para conciliar el sueño, sentimientos de cansancio y tristeza. En concordancia con estos hallazgos, Banerjee y Rai (2020) argumentan que los periodos 
de aislamiento social tienden a generar síntomas de depresión e insomnio en las personas, incluso, implica mayores niveles de ansiedad, estrés crónico y demencia en la adultez tardía. Tal y como describen Torales et al. (2020), la proliferación del COVID-19 ha generado un impacto importante en la salud mental a nivel mundial, y los resultados en este estudio demuestran que Lima Metropolitana no es la excepción.

Con respecto a las comparaciones de acuerdo a variables sociodemográficas, los análisis con respecto al sexo indican que hay diferencias en los niveles de ansiedad entre mujeres y hombres. Particularmente, la diferencia en los niveles de ansiedad fue moderada, siendo mayor en el caso de las mujeres. Además, aunque se encontraran diferencias pequeñas entre ambos sexos en relación a la sintomatología depresiva, las mujeres muestran una mayor prevalencia de dichos indicadores. En consonancia con estos resultados, distintas investigaciones apuntan a que las mujeres presentan mayores niveles de ansiedad y depresión en comparación de los hombres (Brooks et al., 2020; Huarcaya-Victoria, 2020; Özdin \& Özdin, 2020).

Los análisis en base al grupo etario denotan que los jóvenes presentan mayores niveles de ansiedad que los adultos y adultos mayores. Estas diferencias fueron pequeñas y moderadas, respectivamente. Con respecto a la depresión, también se encontraron diferencias moderadas entre los jóvenes y los otros grupos etarios. Estos resultados son similares a los obtenidos en estudios internacionales como en China (Wang et al., 2020) y España (Ozamiz-Etxebarria et al., 2020). Una posible hipótesis establecida por Wang et al. (2020) es que en los jóvenes se presentan más indicadores de ansiedad y depresión porque el periodo de aislamiento irrumpe con sus estudios y su desarrollo profesional.

$\mathrm{Al}$ analizar las diferencias considerando el nivel educativo, los participantes con educación técnica y universitaria incompleta mostraron mayores niveles de ansiedad que los participantes con educación universitaria completa. Estas diferencias fueron pequeñas y moderadas, respectivamente. Los mismos resultados se encontraron para el nivel de depresión, pero las diferencias entre los grupos fueron pequeñas. En congruencia con estudios internacionales, un menor nivel de educación parece estar relacionado con un mayor nivel de depresión y ansiedad (Lei et al., 2020); sobre todo en mujeres (Oliveira et al., 2020; Sinha \& Manna, 2020).

Finalmente, se encontró que las personas que trabajaban o estudiaban de forma virtual mostraron mayores niveles de ansiedad y depresión respecto a las personas que trabajaban de manera presencial, aunque estas diferencias fueron pequeñas. Con respecto a la diferencia en los puntajes de ansiedad, Hilliard et al. (2020) explican que esto puede deberse a la incertidumbre ligada al ambiente de trabajo, sobre todo cuando el trabajo puede ser evaluado. Por otro lado, el mayor nivel de depresión puede deberse al sentimiento de soledad generado por la modalidad virtual (Misirlis et al., 2020) y al aumento de demandas familiares (Cho, 2020).

Como puede apreciarse, los resultados encontrados coinciden con lo reportado en poblaciones distintas durante la pandemia, por ejemplo, poblaciones de India, China, Turquía y España. Se puede concluir, tanto como de estas investigaciones como del trabajo actual, que es necesario y urgente abordar los efectos de la pandemia sobre la salud mental de las personas prestando atención a variables sociodemográficas como el sexo, el nivel educativo y la edad. En este sentido, los resultados implican, en un sentido práctico, la necesidad del reconocimiento de esta problemática por parte de los responsables de las políticas de salud pública; ello con la finalidad de garantizar el acceso a servicios de salud mental de alta calidad. Las limitaciones en los resultados de la presente investigación son consecuencias propias del diseño de este estudio, el cual contribuye a copar con el vacío de conocimiento; mas no verifica modelos teóricos o hipótesis. Por otro lado, la muestra obtenida en el presente estudio no es necesariamente representativa y abarca solo a 
residentes de Lima Metropolitana. Aun así, estos resultados son una aproximación exploratoria al estado de la salud mental a nivel nacional y sientan las bases para investigaciones posteriores o análisis secundarios que puedan generar contribuciones teóricas más amplias a partir de modelos teóricos más definidos, investigaciones con muestras de mayor tamaño o meta-análisis que puedan hacer provecho de los hallazgos reportados en el campo.

\section{Conflicto de intereses}

Los autores del presente artículo declaran no tener ningún conflicto de intereses relacionado a la evaluación y publicación de los resultados encontrados.

\section{Responsabilidad ética}

En esta investigación se evaluaron síntomas de ansiedad y depresión, además, se recopilaron datos sociodemográficos. La información recopilada no permite la identificación de las personas y su uso para fines académicos fue informado y aceptado por los voluntarios. Esto se hizo a través de un consentimiento informado en el que se explicaba el propósito de la investigación y los datos a ser recolectados. Solo los adultos mayores de edad que consintieron el uso de su información forman parte del estudio. La información recolectada fue sistematizada en una base de datos electrónica manejada únicamente por los autores de la investigación. No se cuenta con el aval de un comité de ética. No obstante, los procesos fueron revisados y aprobados por el Comité de Investigación y Ética de la Universidad de Lima, el cual rige la actividad investigadora interna, pero no emite certificados de aprobación para entidades externas.

\section{Contribución de autoría}

DEPM: Contribución en la concepción y diseño del artículo, revisión bibliográfica, redacción y revisión crítica de los resultados para generar la discusión de manera equitativa.
GLAB: Contribución en la concepción y diseño del artículo, revisión bibliográfica, redacción y revisión crítica de los resultados para generar la discusión de manera equitativa.

IDP: Contribución en la concepción y diseño del artículo, revisión bibliográfica, redacción y revisión crítica de los resultados para generar la discusión de manera equitativa.

GLVB: Contribución en la concepción y diseño del artículo, revisión bibliográfica, redacción y revisión crítica de los resultados para generar la discusión de manera equitativa.

LAMS: Contribución en la concepción y diseño del artículo, revisión bibliográfica, redacción y revisión crítica de los resultados para generar la discusión de manera equitativa.

CSLN: Contribución en la concepción y diseño del artículo, revisión bibliográfica, redacción y revisión crítica de los resultados para generar la discusión de manera equitativa.

KAUB: Contribución en la concepción y diseño del artículo, revisión bibliográfica, redacción y revisión crítica de los resultados para generar la discusión de manera equitativa.

ACHZ: Elaboración del método y el análisis estadístico de los datos.

\section{Referencias}

Banerjee, D., \& Rai, M. (2020). Social Isolation in Covid19: The Impact of Loneliness. International Journal of Social Psychiatry, 66(6), 525-527. https://doi.org/ 10.1177\%2F0020764020922269

Brooks, S., Webster, R., Smith, L., Woodland, L., Wessely, S., Greenberg, N., \& Rubin, G. (2020). The Psychological Impact of Quarantine and How to Reduce It: Rapid Review of the Evidence. The Lancet, 395(10227), 912920. https://doi.org/10.1016/S0140-6736(20)30460-8 
Brown, T. A. (2015). Confirmatory Factor Analysis for Applied Research. The Guilford Press.

Cho, E. (2020). Examining Boundaries to Understand the Impact of COVID-19 on Vocational Behaviors. Journal of Vocational Behavior, 119. https://doi.org/10.1016/ j.jvb.2020.103437

Cohen, J. (1992). A Power Primer. Psychological Bulletin, 112(1), 155-1599. https://doi.apa.org/doi/10.1037/00332909.112.1.155

Comisión Económica para América Latina y el Caribe (2020). Dimensionar los efectos del COVID-19 para pensar en la reactivación. Comisión Económica para América Latina y el Caribe. https://repositorio.cepal.org/bitstream/ handle/11362/45445/4/S2000286_es.pdf

Hilliard, J., Kear, K., Donelan, H., \& Heaney, C. (2020). Students' Experiences of Anxiety in an Assessed, Online, Collaborative Project. Computers \& Education, 143. https://doi.org/10.1016/j.compedu.2019.103675

Hossain, M., Sultana, A., \& Purohit, N. (2020). Mental Health Outcomes of Quarantine and Isolation for Infection Prevention: A Systematic Umbrella Review of the Global Evidence. PsyArXiv, 42. https://doi.org/ 10.4178/epih.e2020038

Howitt, D., \& Cramer, D. (2017). Research Methods in Psychology. Pearson.

Huarcaya-Victoria, J. (2020). Consideraciones sobre la salud mental en la pandemia de COVID-19. Revista Peruana de Medicina Experimental y Salud Pública, 37(2), 327334. https://doi.org/10.17843/rpmesp.2020.372.5419

Hyun-Chung, K., So-Young, Y., Bun-Hee, L., So, L., \& Hyoung-Shik, S. (2018). Psychiatric Findings in Suspected and Confirmed Middle East Respiratory Syndrome Patients Quarantined in Hospital: A Retrospective Chart Analysis. Psychiatry Investigation, 15(4), 355-360. https://doi.org/10.30773/ pi.2017.10.25.1

Keith, T. Z. (2019). Multiple regression and beyond: An introduction to multiple regression and structural equation modeling (3rd ed.). Routledge

Lei, L., Huang, X., Zhang, S., Yang, J., Yang, L., \& Xu, M. (2020). Comparison of Prevalence and Associated Factors of Anxiety and Depression Among People
Affected by versus People Unaffected by Quarantine During the COVID-19 Epidemic in Southwestern China. Medical Science Monitor, 26, e924609. https://doi.org/ 10.12659/MSM.924609

Lozano-Vargas, A., \& Vega-Dienstmaier, J. M. (2018). Construcción y propiedades psicométricas de la Escala de Ansiedad de Lima de 20 ítems (EAL-20). Revista de Neuro-Psiquiatría, 81(4), 226-234. https://doi.org/ 10.20453/rnp.v81i4.3437

Misirlis, N., Zwaan, M., \& Weber, D. (2020). International Students' Loneliness, Depression and Stress Levels in COVID-19 Crisis: The Role of Social Media and the Host University. arXiv. https://arxiv.org/ftp/arxiv/ papers/2005/2005.12806.pdf

Oliveira, E., Zeitoune, R., Gallasch, C., Pérez, E., Silva, A., \& Souza, T. (2020). Common Mental Disorders in Nursing Students of the Professionalizing Cycle. Revista Brasileira de Enfermagem, 73(1), e20180154. https://doi.org/10.1590/0034-7167-2018-0154

Organización Mundial de la Salud. (2020, 19 de marzo). Considerations for Quarantine of Individuals in the Context of Containment for Coronavirus Disease (COVID-19): Interim Guidance. https://apps.who.int/ iris/handle/10665/331497

Ozamiz-Etxebarria, N., Dosil-Santamaria, M., PicazaGorrochategui, M., \& Idoiaga-Mondragon, N. (2020). Niveles de estrés, ansiedad y depresión en la primera fase del brote del COVID-19 en una muestra recogida en el norte de España. Cadernos de Saúde Pública, 36(4), e00054020 . http://dx.doi.org/10.1590/0102$311 \times 00054020$

Özdin, S., \& Özdin, S. B. (2020). Levels and Predictors of Anxiety, Depression, and Health Anxiety During COVID-19 Pandemic in Turkish Society: The Importance of Gender. International Journal of Social Psychiatry, 66(5) 504-511. https://doi.org/10.1177\%2F0 020764020927051

Pappa, S., Ntella, V., Giannakas, T., Giannakoulis, V. G., Papoutsi, E., \& Katsaounou, P. (2020). Prevalence of Depression, Anxiety, and Insomnia Among Healthcare Workers During the COVID-19 Pandemic: A Systematic Review and Meta-Analysis. Brain, Behavior, and Immunity, 88, 901-907. https://doi.org/10.1016/ j.bbi.2020.05.026 
Park, S. C., \& Park, Y. C. (2020). Mental Health Care Measures in Response to the 2019 Novel Coronavirus Outbreak in Korea. Psychiatry Investigation, 17(2), 8586. https://doi.org/10.30773/pi.2020.0058

Rubin, G. J., \& Wessely, S. (2020). The Psychological Effects of Quarantining a city. BMJ, 368, m313. https://doi.org/ 10.1136/bmj.m313

Schwartz, J., King, C. C., \& Yen, M. Y. (2020). Protecting Healthcare Workers During the Coronavirus Disease 2019 (COVID-19) Outbreak: Lessons from Taiwan's Severe Acute Respiratory Syndrome Response. Clinical Infectious Diseases, 71(15), 858-890. https://doi.org/ 10.1093/cid/ciaa255

Sinha, D., \& Manna, P. (2020). The Effect of Lockdown Due to Covid-19 on Post-traumatic Stress and Depression Among College Students of Kolkata, Hotspot District of West Bengal, India. Journal of Humanities and Social Science, 25(5), 58-64.

Shigemura, J., Ursano, R., Morganstein, J., Kurosawa, M., \& Benedek, D. M. (2020). Public Responses to The Novel 2019 Coronavirus (2019-nCoV) in Japan: Mental Health Consequences and Target Populations. Psychiatry and Clinical Neurosciences, 74(4), 281-282. https://doi.org/10.1111/pcn.12988

Shuai, L., Yang, L., Zhang, C., Xiang, Y., Liu, Z., Hu, S., \& Zhang, B. (2020). Online Mental Health Services in China During the Covid-19 Outbreak. The Lancet, 7(4), E17-E18. https://doi.org/10.1016/S2215-0366(20)30077-8

Tavares, C. K., Medeiros, P. M. de, Silva, I., Oliveira, V. A. de, Seves, J., Inácio, R., Lima, C. G., \& Rolim, M. L. (2020).
The Emotional Impact of Coronavirus 2019-nCoV (New Coronavirus Disease). Psychiatry Research, 287, 112915. https://doi.org/10.1016/j.psychres.2020.112915

Torales, J., O’Higgins, M., Castaldelli-Maia, J. M., \& Ventriglio, A. (2020). The Outbreak of COVID-19 Coronavirus and its Impact on Global Mental Health. International Journal of Social Psychiatry, 66(4), 317320. https://doi.org/10.1177\%2F0020764020915212

Vega-Dienstmaier, J. M. (2018). Construcción de versiones cortas de la Escala de Psicopatología Depresiva para tamizaje de depresión mayor y sus características psicométricas. Revista de Neuro-Psiquiatría, 81(3), 154-164. https://doi.org/10.20453/rnp.v81i3.3383

Viladrich, C., Angulo-Brunet, A., \& Doval, E. (2017). A Journey Around Alpha and Omega to Estimate Internal Consistency. Anales de Psicología, 33(3), 755-782. https://doi.org/10.6018/analesps.33.3.268401

Wang, C., Pan, R., Wan, X., Tan, Y., Xu, L., Ho, C. S., \& Ho, R. C. (2020). Immediate Psychological Responses and Associated Factors During the Initial Stage of the 2019 Coronavirus Disease (COVID-19) Epidemic Among the General Population in China. International Journal of Environmental Research and Public Health, 17(5), 1729. https://doi.org/10.3390/ijerph17051729

Xiang, Y., Yang, Y., Li, W., Zhang, L., Zhang, Q., Cheung, T., \& Ng, C. H. (2020). Timely Mental Health care for the 2019 Novel Coronavirus Outbreak is Urgently Needed. The Lancet: Psychiatry, 7(3), 228-229. https:/ /doi.org/10.1016/S2215-0366(20)30046-8 
Diego E. Prieto-Molinari

Estudiante de Psicología de la Universidad de Lima, Perú.

ORCID: http://orcid.org/0000-0003-0470-5182

Autor corresponsal: depm_96@outlook.com

Gianella L. Aguirre Bravo

Estudiante de Psicología de la Universidad de Lima, Perú.

ORCID: http://orcid.org/0000-0002-8521-6489

20180023@aloe.ulima.edu.pe

Inés de Pierola

Estudiante de Psicología de la Universidad de Lima, Perú.

ORCID: https://orcid.org/0000-0002-5132-6559

20140403@aloe.ulima.edu.pe

Giancarlo Luna Victoria-de Bona

Estudiante de Psicología de la Universidad de Lima, Perú.

ORCID: https://orcid.org/0000-0002-7711-6429

20173558@aloe.ulima.edu.pe

Lucía A. Merea Silva

Estudiante de Psicología de la Universidad de Lima, Perú.

ORCID: https://orcid.org/0000-0001-7536-5045

20183500@aloe.ulima.edu.pe

Caleb S. Lazarte Nuñez

Estudiante de Psicología de la Universidad de Lima, Perú.

ORCID: https://orcid.org/0000-0001-5211-6687

20191075@aloe.ulima.edu.pe

Karla, A. Uribe-Bravo

Estudiante de Psicología de la Universidad de Lima, Perú.

ORCID: https://orcid.org/0000-0002-7282-4602

20153398@aloe.ulima.edu.pe

Ángel Ch. Zegarra

Licenciado de Psicología por la Universidad de Lima, Perú.

ORCID: http://orcid.org/0000-0001-5873-745X

zegarrapsy@gmail.com 